\title{
Stochastic approach to model spot price and value forward contracts on energy markets under uncertainty
}

\author{
Michał Pawłowski ${ }^{1} \cdot$ Piotr Nowak $^{1}$ (D)
}

Received: 16 December 2020 / Accepted: 5 August 2021 / Published online: 22 August 2021

(c) The Author(s) 2021

\begin{abstract}
The paper deals with a model of electricity spot prices. The proposed dynamics of electricity spot prices is driven by a mean reverting diffusion with jumps having hyperexponential distribution. The analytical formula for the forward contract's price is derived in a crisp case. Inasmuch as the model parameters are considered to be evaluated imprecisely, their fuzzy counterparts are introduced. With usage of the fuzzy arithmetic, the analytical expression for the forward contract's price is derived. Several numerical examples highlighting attributes of the fuzzy forward electricity prices are brought out.
\end{abstract}

Keywords Electricity markets $\cdot$ Fuzzy set theory $\cdot$ Jump-diffusion $\cdot$ Pricing $\cdot$ Decision-making

\section{Introduction}

The liberalization of electricity markets in Europe was a process which started in the early 1990s. Before, all market activities were performed by state-owned monopolists. The establishment of the internal market in electricity in many countries was a consequence of an implementation of the Directive 96/92/EC by the European Union on 19th December 1996. The aim of the new legislation was to open the electricity market to free competition accompanied by the unbundling of the sector. Moreover, there was a need to develop electricity production, transportation and distribution sectors, simultaneously enlarging security of supply and participation of renewable energy sources in the market. These aspects were usually involved with the necessity to create a power exchange in the country which was initiating the changes.

The time series of electricity spot prices (related to a dayahead market), in contradiction to, e.g., share market's prices paths, is characterized by existence of weekly and yearly seasonality to which the price process immediately reverts after abrupt jumps of prices. These sudden spikes may be

Piotr Nowak

pnowak@ibspan.waw.pl

Michał Pawłowski

m.p.k.pawlowski@gmail.com

1 Systems Research Institute, Polish Academy of Sciences, Newelska 6, 01-447 Warsaw, Poland due to failure of a transmission network or a power plant, rapid temperature change, power losses, turning off power blocks, low levels of water or droughts, changing possibilities of exploitation of renewable energy sources (wind and photovoltaic generations are variable), etc. - combined with inelasticity of supply and demand (since electricity cannot be effectively stored).

In 1973, Black and Scholes derived the European option pricing formula, using a geometric Brownian motion for description of dynamics of the underlying asset prices. However, the Black-Scholes approach suffers some drawbacks and therefore many alternatives to it have been proposed. Among them Levy processes have been proposed to describe log-prices of underlying assets, including the mixed-exponential jump-diffusion (MEM) of Cai and Kou (2011) and the models of Barndorff-Nielsen (1998); Madan and Seneta (1990). In Nowak (2011); Nowak and Romaniuk (2014) Levy jump-diffusions and various sources of uncertainty on the financial market were considered, using semimartingale characteristics, see e.g. Nowak (2002). Markov-modulated Levy processes were used by Deelstra and Simon (2017) for the valuation of exchange options and quanto options. Bao and Zhao (2019) valued vanilla, binary, and exchange options, whose underlying assets are driven by Markovian regime switching exponential Levy processes and interest rates are modelled by Markovian regime switching Hull-White process. Feng et al. (2020) considered the European option pricing model with pure jumps, assuming that volatility and interest rate 
are driven by the CIR processes. The Vasicek stochastic interest rate model and a general Levy process, describing the underlying asset, were studied by Tan et al. (2020), who obtained the options pricing formulas under the equivalent martingale measure. This approach includes the jump-diffusion model and the infinite activities Levy model as special cases.

Non-fuzzy financial models usually make use of classical statistical methods of estimation of parameters. However, markets dynamically fluctuate, in particular it refers to exceptionally volatile energy markets. Therefore, crisp financial models are complemented by their fuzzy counterparts and estimation methods are substituted by expert knowledge who can judge based on market fundamentals. This is why the motivation of our work is to create a model of dynamics of electricity spot prices and valuation of forward contracts in a fuzzy environment. Thus we propose a crisp stochastic model which is realistic and enables to compute the forward price analytically. Additionally, we would like to use a method of pricing which takes into consideration hidden quantities, that is, the market prices of risk. Simultaneously, the existing crisp analytical formula will enable the valuation of forward contracts also in the fuzzy case with application of the fuzzy arithmetic.

In the paper, a mean-reverting jump-diffusion process of electrical energy spot prices is proposed. The distinctive properties of the energy prices' time series are taken into consideration. Our model belongs to the class of one-factor continuous-time models which are characterized by good adaptivity to data, existence, not infrequently, of analytical solutions to numerous provided issues. In practice, it is often impossible to use statistical estimation of the market and model parameters and obtain them in the crisp form, since the market fluctuates (see, e.g. Wu 2004). The mentioned type of uncertainty is modelled in the paper by fuzzy numbers. Thus, we combine a stochastic and fuzzy approach in the proposed pricing method.

The summary of main contributions of the paper is presented below.

- We propose to choose the hyperexponential jump-size distribution, since this distribution can approximate distributions from a large class, including the long-tailed Pareto and Weibull, arbitrarily closely, see Feldmann and Whitt (1998).

- The complete derivation of the analytical formula for the forward price which allows for pricing of forward contracts in a crisp case. To derive this formula, we use the Esscher transformed equivalent probability measure, which requires application of advanced stochastic methods.

- Obtaining the analytical expression for the forward contract's price in the fuzzy environment.
- Featuring quantitative properties of the fuzzy forward contracts by providing numerical examples with real-life parameters values applying a proposed method of calibrating the market prices of risk to the observed prices on the market.

- Conceptualization of the decision-making algorithm applied for the energy forward contracts enriched by real-life case studies.

\section{State of art}

\subsection{Electricity spot prices models}

The basic, initial model of the spot prices evolution was introduced by Lucia and Schwartz (2002) in which the authors decomposed the signal to a seasonality and a meanreverting to zero diffusion process. However, they did not take the possibility of jumps in prices into account.

Cartea and Figueroa (2005) introduced a mean-reverting jump-diffusion model with normally distributed jumps. There is a possibility of deriving the analytical formula for the forward price. Unfortunately, the jump-size distribution very rarely is normal.

An interesting approach was presented in a threshold model of Geman and Roncoroni (2006) where the meanreverting diffusion was combined with a time-inhomogeneous Poisson process of a truncated exponential distribution of jumps. Moreover, to allow for downward, reverting to the mean jumps, the authors introduced a characteristic function indicating a sign of a jump which depends on a current value of a price. The proposed switching threshold is a constant positive spread over a seasonality. Notwithstanding, the model has some drawbacks as well. Benth et al. (2011) criticizes the choice of the truncated exponential distribution because of the fact that jumps higher than some predefined threshold are disallowed. There is also noted that two consecutive jumps of the same sign are impossible to occur and after estimating the model's parameters the magnitude of the speed of mean-reversion appears to be inadequate simultaneously for both the base and jump regimes.

Another interesting subclass of one-factor models are regime-switching models. They are very popular nowadays and also in the field of electrical energy prices modelling they are widely applicable. The reason is that one can define separate forms of dynamics for all substantially different ranges of prices values, usually there are three of them: two spike regimes when the prices achieve anomalous values after the upward and downward jumps, and a normal, base regime. There is also a transition matrix which links the regimes by indicating how much the transition from one state to another is probable. Estimation of regime-switching models' parameters is not straightforward, inasmuch as 
regimes are latent variables (not observable directly). For details, see Janczura and Weron (2010); de Jong and Huisman (2002); Lindstrom and Regland (2012).

An alternative to all above-mentioned approaches may be a model in which a diffusion generated by a Wiener process is superseded by very frequent and small jumps (representing typical, daily movements of prices) generated by a Levy process of infinite activity. The Levy process is also responsible for big jumps in prices (substitution for the Poisson process). The model was described by Benth and Saltyte-Benth (2004). It must be noted that estimation of the parameters of $\alpha$-stable non-Gaussian or generalized hyperbolic distributions is cumbersome and requires sophisticated numerical methods, because there is no closed-form formulas for the density and distribution functions.

There is no possibility to obtain the analytical formula for the forward price in the latter three models. We consider it as a big disadvantage, inasmuch as this formula not only allows for a precise valuation of any forward or futures contract, which are the most popular energy derivatives, but also it is a basis for calibration of the model to observed prices on the market.

A significant feature that should be expressed by an electricity price dynamics model is the commonly noticed inverse leverage effect, see, e.g. Nomikos and Soldatos (2010). That is, with bigger prices, the supply stack curve becomes more sloped, causing relatively higher price changes and greater volatility, which in turn "widens" the right tail of the spot price log-returns distribution. As a result, the distribution is skewed to the right, and the right tail is heavier compared to the log-normal distribution.

A recent comparison of electricity spot price modelling for pricing of derivatives and risk management was performed by Canakoglu and Adiyeke (2020).

\subsection{Pricing derivatives in a fuzzy environment}

Recently, the literature concerning financial derivatives valuation in a fuzzy environment has been developed.

A fuzzy approach, similar to the one proposed in this paper, to European options pricing for the traditional Black-Scholes model one can find in Wu (2004). Fuzzy estimation of the volatility in the Black-Scholes model was introduced by Chrysafis and Papadopoulos (2009). Thiagarajah et al. (2007) used adaptive fuzzy numbers in the Black-Scholes setting. The paper of Xian-Dong and Jian-Min (2014) was devoted to a fuzzy reload option pricing problem. Another method of European option pricing was presented by Yoshida (2003), where the rational expected option price depending on a fuzzy goal was applied. Zhang et al. (2015) applied the stochastic and fuzzy approach to the valuation of geometric Asian options. An improvement of the fuzzy version of the Black-Scholes option pricing formula using triangular approximations was proposed by de Andres-Sanchez (2018). The papers mentioned above concern the case, where a geometric Brownian motion drives the underlying asset.

The approach of Wu (2004) was further extended and developed by Nowak and Pawłowski $(2017,2019)$; Nowak and Romaniuk (2010, 2013b, 2014) to the case, where a Levy process with jumps describes the primary financial instrument. Applications of some jump-diffusion models were discussed by Liu and Li (2013); Xu et al. (2013); Zhang et al. (2012). A geometric Levy process was used by Wang and Hea (2016) for $\mathrm{n}$-fold compound option pricing in the fuzzy framework. The same stochastic process was applied by Wu et al. (2017) to price the total return swap, which is a credit derivative. The problem of European options pricing in a fuzzy environment, based on an infinite pure jump Levy process, was discussed by Zhang and Watada (2018a, b).

Combining the stochastic and fuzzy methods, Biancardi and Villani (2017) valued the R\&D compound option. Tolga (2017) used Type-2 fuzzy logic to real option valuation.

Worth mentioning is also a multiperiod binomial model for options pricing in a vague world, considered by Muzzioli and Torricelli (2004). In turn, Zmeskal (2010) proposed a hybrid fuzzy-stochastic binomial model for pricing the American real option. Finally, Anzilli et al. (2018) dealt with the valuation of the minimum guarantee option embedded in equity-linked life insurance, applying fuzzy binomial-tree model for the description of the asset price evolution.

Some new option pricing methods in fuzzy environment, using advanced techniques of fuzzy mathematics, have been recently introduced. In this context, it is worth noticing a methodology for option pricing, applied in Li et al. (2018), where a nonlinear fuzzy-parameter PDE model is built for obtaining option prices and dominating optimal hedging strategies are developed. Qin et al. (2020) considered the fractional Brownian motion to model the stock price in the binary option pricing problem, taking into account the long memory property of financial markets. Furthermore, a new approach to valuation of portfolio and options in the Black-Scholes model in fuzzy environment, involving fuzzy geometric Brownian motion, fuzzy lognormal distribution, as well as fuzzy Ito integral, is discussed by Dash et al. (2021).

To the authors' best knowledge, the problem of pricing forward contracts on the electricity market, with an application of a mean-reverting jump-diffusion to the description of the underlying asset, has not been studied in a fuzzy environment yet. 


\section{Dynamics of electricity spot prices within the model}

In this section dynamics of our model is presented. The idea of the hyperexponential distribution is drawn from Feldmann and Whitt (1998) (where the authors analyze network performance models). In contrast to modelling equities' prices, applying the hyperexponential jump-size distribution to the mean-reverting jump-diffusion process of the dynamics of electricity prices is novel. This distribution has a distinctive property that it can approximate distributions from a large class, including the long-tailed Pareto and Weibull, arbitrarily closely.

Let $\mathcal{T}=\left[0, T^{*}\right], T^{*}>0$ be a finite time horizon. Let $\left(\Omega, \mathcal{F},\left(\mathcal{F}_{t}\right)_{t \in \mathcal{T}}, \mathbb{P}\right)$ be a filtered probability space, satisfying the usual assumptions, on which all the below used processes and random variables are defined.

$\Omega$ represents the set of scenarios that can occur in the financial market, $\sigma$-algebra $\mathcal{F}$ of subsets of $\Omega$ contains all possible events, and probability measure $\mathbb{P}: \mathcal{F} \rightarrow[0,1]$ assigns a probability belonging to $[0,1]$ to each event. Finally, filtration $\left(\mathcal{F}_{t}\right)_{t \in \mathcal{T}}$ is an increasing family of $\sigma$-algebras included in $\mathcal{F}$. For each $t \in \mathcal{T}, \sigma$-algebra $\mathcal{F}_{t}$ is interpreted as the information known at time $t$.

The spot price process $S_{t}$ is composed of the following constituents:

$S_{t}=\exp \left(g(t)+X_{t}\right)$,

$d X_{t}=-\mu X_{t} d t+\sigma d W_{t}+d J_{t}$,

where $g(t)$ is a deterministic seasonality estimated from historical data, $\mu$ and $\sigma$ are positive constants, $\left(W_{t}\right)_{t \in \mathcal{T}}$ is a $(\mathcal{F}, \mathbb{P})$ Wiener process, $\left(J_{t}\right)_{t \in \mathcal{T}}$ is a $(\mathcal{F}, \mathbb{P})$ compound Poisson process of the form $J_{t}=\sum_{i=1}^{N_{t}} Z_{i}, t \in \mathcal{T}$, where $N_{t}$ is a Poisson process with constant intensity $\lambda,\left\{Z_{i}\right\}_{i \in \mathbb{N}}$ are i.i.d. jump magnitudes of the hyperexponential distribution, i.e. with density

$f(z)=\sum_{i=1}^{n} p_{i} \xi_{i} \exp \left(-\xi_{i} z\right)$

where $p_{i} \geq 0, \sum_{i=1}^{n} p_{i}=1, \xi_{i}>0, z \geq 0$.

From Ito's lemma, it follows that $S_{t}$ is described by the stochastic differential equation

$d S_{t}=\mu\left(\rho(t)-\ln S_{t}\right) S_{t} d t+\sigma S_{t} d W_{t}+S_{t}\left(e^{Z_{N_{t}}}-1\right) d N_{t}$,

where $\rho(t)=\frac{1}{\mu}\left(\frac{d g(t)}{d t}+\frac{1}{2} \sigma^{2}\right)+g(t), Z_{0}=0$.

$\left(\mathcal{F}_{t}\right)_{t \in \mathcal{T}}$ is generated by $W$ and $J$ and augmented to encompass $\mathbb{P}$-null sets from the $\sigma$-field $\mathcal{F}=\mathcal{F}_{T^{*}}$.

\section{Pricing forward contracts with crisp parameters}

Let $\left(I_{t}\right)_{t \in \mathcal{T}}$ be an independent increments process with semimartingale characteristics $(\gamma, C, l(d u, d z))$ under $\mathbb{P}$. The riskneutral measure $\mathbb{Q}$, equivalent to $\mathbb{P}$, is given by the Esscher transform, i.e.

$\left.\frac{d \mathbb{Q}}{d \mathbb{P}}\right|_{\mathcal{F}_{t}}=\hat{Z}^{\theta}(t) \bar{Z}^{\theta}(t)$,

where $\theta(t)=(\hat{\theta}(t), \bar{\theta}(t))$ is a 2-dimensional vector of $\mathbb{R}$-valued continuous functions on $\mathcal{T}$,

$\hat{Z}^{\theta}(t)=\exp \left(\int_{0}^{t} \hat{\theta}(s) d W_{s}-\frac{1}{2} \int_{0}^{t} \hat{\theta}^{2}(s) d s\right)$,

$\bar{Z}^{\theta}(t)=\exp \left(\int_{0}^{t} \bar{\theta}(s) d I_{s}-\phi(0, t, \bar{\theta}(\cdot))\right)$,

$\sup _{t \in \mathcal{T}}|\bar{\theta}(t)| \leq c$ and $\int_{\mathcal{T}} \int_{1}^{\infty}\left\{e^{c z}-1\right\} l(d z, d u)<\infty$

for some $c \in \mathbb{R}_{+}$, and

$$
\begin{gathered}
\phi(0, t, \bar{\theta}(\cdot))=\int_{0}^{t} \bar{\theta}(u) d \gamma(u)+\frac{1}{2} \int_{0}^{t} \bar{\theta}^{2}(u) d C(u) \\
+\int_{0}^{t} \int_{\mathbb{R}}\left\{e^{\bar{\theta}(u) z}-1-\bar{\theta}(u) z \mathbb{\mathbb { 1 }}_{|z|<1}\right\} l(d z, d u) .
\end{gathered}
$$

The equivalence of $\mathbb{P}$ and $\mathbb{Q}$ on $(\Omega, \mathcal{F})$ means that impossible events defined by them are the same, i.e.

$\mathbb{P}(A)=0 \Longleftrightarrow \mathbb{Q}(A)=0, \quad A \in \mathcal{F}$.

We will use the following proposition proved by Benth and Sgarra (2012) in a more general case.

Proposition 1 Process $W_{t}^{\mathbb{Q}}=W_{t}-\int_{0}^{t} \hat{\theta}(s) d s, \quad t \in \mathcal{T}$, is $a \mathbb{Q}$ -Brownian motion and with respect to $\mathbb{Q}$ the independent increments process I has drift

$\gamma(t)+\int_{0}^{t} \int_{|z|<1} z\left\{e^{\bar{\theta}(u) z}-1\right\} l(d z, d u)+\int_{0}^{t} \bar{\theta}^{2}(u) d C(u)$

and predictable compensator measure $e^{\bar{\theta}(t) z} l(d z, d t)$.

Definition 1 (Forward price) A forward price $F^{\mathbb{Q}}(t, T)$ at time $t$ is a conditional expected value, with respect to the risk-neutral measure $\mathbb{Q}$, of a spot price in the future time $T$ :

$F^{\mathbb{Q}}(t, T)=\mathbb{E}^{\mathbb{Q}}\left[S_{T} \mid \mathcal{F}_{t}\right]$.

In this paper we assume that the risk-neutral measure is given by the Esscher transform (5) for processes $W$ and $I=J$, under assumptions that $\hat{\theta} \equiv \hat{\theta}_{0} \in \mathbb{R}, \bar{\theta} \equiv \bar{\theta}_{0} \in \mathbb{R}$, 
usually called the market price of diffusion risk and the market price of jump risk, respectively, and that $\max \left(\bar{\theta}_{0}+1,\left|\bar{\theta}_{0}\right|\right)<\min _{1 \leq i \leq n} \xi_{i}$.

Theorem 1 The forward price in a crisp case within the model defined in "Dynamics of electricity spot prices within the model" by (1,2 and 3) is described by the following analytical formula:

$F^{\mathbb{Q}}(t, T)=\mathbb{E}^{\mathbb{Q}}\left[S_{T} \mid \mathcal{F}_{t}\right]=e^{g(T)}\left(\frac{S_{t}}{e^{g(t)}}\right)^{m_{t, T}}$.
$\exp \left[\frac{\sigma\left(1-m_{t, T}\right)\left(\sigma\left(1+m_{t, T}\right)+4 \hat{\theta}_{0}\right)+4 \lambda^{\mathbb{Q}} \sum_{i=1}^{n} p_{i}^{\mathbb{Q}} \ln \frac{\xi_{i}^{\mathbb{Q}}-m_{t, T}}{\xi_{i}^{\mathbb{Q}}-1}}{4 \mu}\right], t \in \mathcal{T}$,

where $m_{t, T}=e^{-\mu(T-t)}, \quad \xi_{i}^{\mathbb{Q}}=\xi_{i}-\bar{\theta}_{0}, \quad p_{i}^{\mathbb{Q}}=\frac{p_{i} \frac{\xi_{i}}{\xi_{i}^{\mathbb{Q}}}}{\sum_{i=1}^{n} p_{i} \frac{\xi_{i}}{\xi_{i}^{\mathbb{Q}}}}$, and $\lambda^{\mathbb{Q}}=\lambda \sum_{i=1}^{n} p_{i} \frac{\xi_{i}}{\xi_{i}^{\mathbb{Q}}}$.

Proof Let us revise the Eq. (4). We use Ito's lemma for the process $Y_{t}=\ln \left(S_{t}\right)$ and change the physical measure $\mathbb{P}$ to the equivalent risk-neutral measure $\mathbb{Q}$, given by the Esscher transform. Applying Proposition 1, we obtain

$d Y_{t}=\mu\left(\rho^{\mathbb{Q}}(t)-Y_{t}\right) d t+\sigma d W_{t}^{\mathbb{Q}}+Z_{N_{t}^{\mathbb{Q}}}^{\mathbb{Q}} d N_{t}^{\mathbb{Q}}$,

where $\rho^{\mathbb{Q}}(t)=\frac{1}{\mu} \frac{d g(t)}{d t}+g(t)+\frac{\sigma \hat{\theta}_{0}}{\mu}, N^{\mathbb{Q}}$ is a $\mathbb{Q}$-Poisson process with the intensity $\lambda^{\mathbb{Q}}=\lambda \int_{\mathbb{R}} e^{\bar{\theta}_{0} z} f(z) d z=\lambda \sum_{i=1}^{n} p_{i} \frac{\xi_{i}}{\xi_{i}^{\mathbb{Q}}}$, independent from $W^{\mathbb{Q}}$, and $\left\{Z_{i}^{\mathbb{Q}}\right\}_{i \in \mathbb{N}}$ are independent identically distributed random variables with the hyperexponential distribution with respect to $\mathbb{Q}$, having density

$f^{\mathbb{Q}}(z)=\frac{e^{\bar{\theta}_{0} z} f(z)}{\int_{\mathbb{R}} e^{\bar{\theta}_{0} z} f(z) d z}=\sum_{i=1}^{n} p_{i}^{\mathbb{Q}} \xi_{i}^{\mathbb{Q}} \exp \left(-\xi_{i}^{\mathbb{Q}} z\right), z \geq 0$.

After multiplying both sides of (8) by $m_{t, T}$ and integrating from $t$ to $T$, the equation converts to

$$
\begin{aligned}
& \int_{t}^{T} m_{s, T} d Y_{s}=\int_{t}^{T} m_{s, T} d g(s)+\int_{t}^{T} \mu m_{s, T} g(s) d s \\
& \quad-\int_{t}^{T} \mu m_{s, T} Y_{s} d s \\
& \quad+\int_{t}^{T} \sigma \hat{\theta}_{0} m_{s, T} d s+\int_{t}^{T} \sigma m_{s, T} d W_{s}^{\mathbb{Q}}+\int_{t}^{T} m_{s, T} Z_{N_{s}^{\mathbb{Q}}}^{\mathbb{Q}} d N_{s}^{\mathbb{Q}} .
\end{aligned}
$$

$-\int_{t}^{T} \mu m_{s, T} Y_{s} d s=m_{t, T} Y_{t}-Y_{T}+\int_{t}^{T} m_{s, T} d Y_{s}$

and

$$
\int_{t}^{T} \mu m_{s, T} g(s) d s=g(T)-m_{t, T} g(t)-\int_{t}^{T} m_{s, T} d g(s),
$$

we may write

$$
\begin{aligned}
Y_{T} & =g(T)+\left(Y_{t}-g(t)\right) m_{t, T}+\int_{t}^{T} \sigma \hat{\theta}_{0} m_{s, T} d s \\
& +\int_{t}^{T} \sigma m_{s, T} d W_{s}^{\mathbb{Q}} \\
& +\int_{t}^{T} m_{s, T} Z_{N_{s}^{\mathbb{Q}}}^{\mathbb{Q}} d N_{s}^{\mathbb{Q}} .
\end{aligned}
$$

Applying the Dynkin lemma and a technique similar as in Nowak and Romaniuk (2013a), we obtain the following equality

$$
\begin{aligned}
\mathbb{E}^{\mathbb{Q}}\left[\operatorname { e x p } \left(\int_{t}^{T} \sigma m_{s, T} d W_{s}^{\mathbb{Q}}\right.\right. \\
\left.\left.+\int_{t}^{T} m_{s, T} Z_{N_{s}^{\mathbb{Q}}}^{\mathbb{Q}} d N_{s}^{\mathbb{Q}}\right) \mid \mathcal{F}_{t}\right] \\
=\mathbb{E}^{\mathbb{Q}}\left[\exp \left(\int_{t}^{T} \sigma m_{s, T} d W_{s}^{\mathbb{Q}}\right) \mid \mathcal{F}_{t}\right] \\
\mathbb{E}^{\mathbb{Q}}\left[\left.\exp \left(\int_{t}^{T} m_{s, T} Z_{N_{s}^{\mathbb{Q}}}^{\mathbb{Q}} d N_{s}^{\mathbb{Q}}\right)\right|_{\left.\mathcal{F}_{t}\right] .}\right.
\end{aligned}
$$

Using $(13,14)$, the fact that $S_{T}=e^{Y_{T}}$ and denoting $G(t)=e^{g(t)}$,

Because 


$$
\begin{aligned}
& F^{\mathbb{Q}}(t, T)=\mathbb{E}^{\mathbb{Q}}\left[S_{T} \mid \mathcal{F}_{t}\right] \\
& =G(T)\left(\frac{S_{t}}{G(t)}\right)^{m_{t, T}} \exp \left(\int_{t}^{T} \sigma \hat{\theta}_{0} m_{s, T} d s\right) . \\
& \mathbb{E}^{\mathbb{Q}}\left[\exp \left(\int_{t}^{T} \sigma m_{s, T} d W_{s}^{\mathbb{Q}}\right) \mid \mathcal{F}_{t}\right] \\
& \mathbb{E}^{\mathbb{Q}}\left[\exp \left(\int_{t}^{T} m_{s, T} Z_{N_{s}^{\mathbb{Q}}}^{\mathbb{Q}} d N_{s}^{\mathbb{Q}}\right) \mid \mathcal{F}_{t}\right] \\
& =G(T)\left(\frac{S_{t}}{G(t)}\right)^{m_{t, T}} \exp \left(\int_{t}^{T} \sigma m_{s, T}\left(\frac{1}{2} \sigma m_{s, T}+\hat{\theta}_{0}\right) d s\right) \text {. } \\
& \mathbb{E}^{\mathbb{Q}}\left[\exp \left(\int_{t}^{T} m_{s, T} Z_{N_{s}^{\mathbb{Q}}}^{\mathbb{Q}} d N_{s}^{\mathbb{Q}}\right) \mid \mathcal{F}_{t}\right] \\
& =G(T)\left(\frac{S_{t}}{G(t)}\right)^{m_{t, T}} \\
& \exp \left(\frac{\sigma\left(1-m_{t, T}\right)}{4 \mu}\left(\sigma\left(1+m_{t, T}\right)+4 \hat{\theta}_{0}\right)\right) \text {. } \\
& \mathbb{E}^{\mathbb{Q}}\left[\exp \left(\int_{t}^{T} m_{s, T} Z_{N_{s}^{\mathbb{Q}}}^{\mathbb{Q}} d N_{s}^{\mathbb{Q}}\right) \mid \mathcal{F}_{t}\right]
\end{aligned}
$$

inasmuch as

$$
\begin{aligned}
\mathbb{E}^{\mathbb{Q}}\left[\exp \left(\int_{t}^{T} \sigma m_{s, T} d W_{s}^{\mathbb{Q}}\right) \mid \mathcal{F}_{t}\right] \\
=\exp \left(\frac{1}{2} \int_{t}^{T} \sigma^{2} e^{-2 \mu(T-s)} d s\right) .
\end{aligned}
$$

Following the considerations of Cartea and Figueroa (2005) (part A of Appendix), we may write

$$
\begin{aligned}
\mathbb{E}^{\mathbb{Q}}\left[\exp \left(\int_{t}^{T} m_{s, T} Z_{N_{s}^{\mathbb{Q}}}^{\mathbb{Q}} d N_{s}^{\mathbb{Q}}\right) \mid \mathcal{F}_{t}\right] \\
=\exp \left(\int_{t}^{T}\left(\mathbb{E}^{\mathbb{Q}}\left[e^{m_{s, T} Z_{N_{s}^{\mathbb{Q}}}^{\mathbb{Q}}}\right]-1\right) \lambda^{\mathbb{Q}} d s\right) .
\end{aligned}
$$

The latter exponent may be explicitly calculated. For this purpose, first of all we calculate

$\mathbb{E}^{\mathbb{Q}}\left[e^{m_{s, T} Z_{N_{s}^{\mathbb{Q}}}^{\mathbb{Q}}}\right]$
The straightforward calculation yields

$\mathbb{E}^{\mathbb{Q}}\left[e^{m_{s, T} Z_{N_{s}^{\mathbb{Q}}}^{\mathbb{Q}}}\right]=\sum_{i=1}^{n} p_{i}^{\mathbb{Q}} \frac{\xi_{i}^{\mathbb{Q}} e^{\mu(T-s)}}{\xi_{i}^{\mathbb{Q}} e^{\mu(T-s)}-1}$.

Thus,

$$
\begin{aligned}
& \mathbb{E}^{\mathbb{Q}}\left[\exp \left(\int_{t}^{T} m_{s, T} Z_{N_{s}^{\mathbb{Q}}}^{\mathbb{Q}} d N_{s}^{\mathbb{Q}}\right) \mid \mathcal{F}_{t}\right] \\
& =\exp \left[\int \sum_{t}^{T} p_{i}^{\mathbb{Q}} \frac{\xi_{i}^{\mathbb{Q}} e^{\mu(T-s)}}{\xi_{i}^{\mathbb{Q}} e^{\mu(T-s)}-1} \lambda^{\mathbb{Q}} d s-\lambda^{\mathbb{Q}}(T-t)\right] \\
& =\exp \left[\frac{\lambda^{\mathbb{Q}}}{\mu} \sum_{i=1}^{n} p_{i}^{\mathbb{Q}} \ln \frac{\xi_{i}^{\mathbb{Q}}-m_{t, T}}{\xi_{i}^{\mathbb{Q}}-1}\right],
\end{aligned}
$$

which finishes the proof.

Definition 2 (Forward contract) A forward contract with delivery of electricity during days $T_{1}<\ldots<T_{N} \in \mathcal{T}$ and a delivery price $K$ is a derivative instrument which enables to receive electricity on delivery days for $K$, therefore financially (for a purpose of pricing) it pays the difference

$\frac{1}{N} \sum_{i=1}^{N} S_{T_{i}}-K$

Let $F_{t}^{\mathbb{Q}}$ denote the value of this forward contract. Thanks to Theorem 1, pricing of forward contracts is made analytically. To this end, we calculate

$F_{t}^{\mathbb{Q}}=\mathbb{E}^{\mathbb{Q}}\left[\frac{1}{N} \sum_{i=1}^{N} S_{T_{i}}-K \mid \mathcal{F}_{t}\right]=\frac{1}{N} \sum_{i=1}^{N} F^{\mathbb{Q}}\left(t, T_{i}\right)-K$

and for each element of the sum we adapt the formula (7).

Definition 3 (Forward price of a forward contract) A forward price $K_{t}$ of a forward contract calculated at time $t$ is such a value of a delivery price $K$ introduced in Definition 2, that $F_{t}^{\mathbb{Q}}=\frac{1}{N} \sum_{i=1}^{N} F^{\mathbb{Q}}\left(t, T_{i}\right)-K_{t}=0$.

\section{Pricing forward contracts with fuzzy parameters}

In this section, we derive the fuzzy version of the forward price. Some details concerning fuzzy numbers as well as fuzzy and interval arithmetic can be found in Nowak and Pawłowski (2017); Wu (2004). We introduce necessary notations. 
We use symbols $\mathbb{R}, \mathcal{B}(\mathbb{R})$, and $\mathbb{F}(\mathbb{R})$ to denote the set of real numbers, the $\sigma$-field of Borel subsets of $\mathbb{R}$, and the set of fuzzy numbers, respectively.

Let $\tilde{a} \in \mathbb{F}(\mathbb{R})$. We denote by $\mu_{\tilde{a}}: \mathbb{R} \rightarrow[0,1]$ its membership function. Its $\alpha$-level sets, for arbitrary $\alpha \in[0,1]$, are denoted by $\tilde{a}_{\alpha}=\left[\tilde{a}_{\alpha}^{L}, \tilde{a}_{\alpha}^{U}\right]$, where $-\infty<\tilde{a}_{\alpha}^{L} \leq \tilde{a}_{\alpha}^{U}<\infty$.

Let $L, R:[0,1] \rightarrow[0,1]$ be continuous and strictly decreasing functions such that $L(0)=R(0)=1$, $L(1)=R(1)=0$ and let $a_{1}, a_{2}, a_{3} \in \mathbb{R}$ satisfy the inequality: $a_{1}<a_{2}<a_{3} . \tilde{a} \in \mathbb{F}(\mathbb{R})$ is called an L-R (left-right) fuzzy number if its membership function has the form:

$\mu_{\tilde{a}}(x)=\left\{\begin{array}{ll}L\left(\frac{a_{2}-x}{a_{2}-a_{1}}\right) & \text { for } a_{1} \leq x \leq a_{2} \\ R\left(\frac{x-a_{2}}{a_{3}-a_{2}}\right) & \text { for } a_{2} \leq x \leq a_{3} \\ 0 & \text { otherwise }\end{array}\right.$.

In particular, $\tilde{a}$ is called a triangular fuzzy number (denoted by $\left.\tilde{a}=\left(a_{1}, a_{2}, a_{3}\right)\right)$ if $L(y)=R(y)=1-y$.

Let $(\Omega, \mathcal{F})$ be a measurable space. A function $\tilde{X}: \Omega \mapsto \mathbb{F}(\mathbb{R})$ is a fuzzy random variable (see, e.g. Puri and Ralescu 1986) if for each $\alpha \in[0,1]$

$\{(\omega, x): \tilde{X}(\omega)(x) \geq \alpha\} \in \mathcal{F} \times \mathcal{B}(\mathbb{R})$.

We denote by $\oplus, \ominus, \otimes$ and $\oslash$ the arithmetic operations between fuzzy numbers, defined with application of the Extension Principle (see, e.g. Zadeh 1975a, b, c) and corresponding standard arithmetic operations,,$+- \times$, between real numbers, respectively.

Moreover, we use symbols $\oplus_{\text {int }}, \ominus_{\text {int }}, \otimes_{\text {int }}$ and $\oslash_{\text {int }}$ to denote the arithmetic operations between closed intervals.

As it was presented by Nowak and Pawłowski (2017); $\mathrm{Wu}$ (2004), $\alpha$-level sets of results of fuzzy arithmetic operations between fuzzy arguments correspond to interval arithmetic operations between $\alpha$-level sets of these arguments.

Since the parameters of the model considered in this paper are uncertain, we assume that they have the form of L-R fuzzy numbers. Their values can be obtained i.a. using expert knowledge (see, e.g. Buckley and Eslami 2007; GilLafuente 2005; Nowak and Pawłowski 2017). The symbol , used above fuzzy parameters in the following part of this paper, indicates their fuzziness.

A similar approach to pricing in a fuzzy environment was the first time used by Wu (2004) in the case of European options.

The model parameters $\mu, \sigma, \lambda, \xi=\left\{\xi_{i}\right\}_{i=1}^{n}$ are replaced by their L-R fuzzy counterparts $\tilde{\mu}, \tilde{\sigma}, \tilde{\bar{\lambda}}, \tilde{\xi}=\left\{\tilde{\xi}_{i}\right\}_{i=1}^{n}$. For each $t \in \mathcal{T}$, the value of the process $\tilde{S}_{t}$ has the form of a fuzzy random variable. We assume that $\tilde{\mu}, \tilde{\sigma}, \tilde{\lambda}$, $\tilde{\xi}_{1} \ominus \bar{\theta}_{0} \ominus 1, \tilde{\xi}_{2} \ominus \bar{\theta}_{0} \ominus 1, \ldots, \tilde{\xi}_{n} \ominus \bar{\theta}_{0} \ominus 1$, and $\tilde{S}_{t}, t \in \mathcal{T}$, are positive, i.e. their membership functions are positive only for positive arguments.
Let $\Sigma$ be the set of symbols $\Sigma=\{L, U\}$ and ${ }^{\prime}: \Sigma \rightarrow \Sigma$ the operator given by: $L^{\prime}=U, U^{\prime}=L$.

Theorem 2 The fuzzy forward price within the model defined in "Dynamics of electricity spot prices within the model" by (1,2 and 3$)$ is described by the following analytical formula:

$$
\begin{aligned}
& \tilde{F}^{\mathbb{Q}}(t, T) \\
& =\exp \left[\tilde{m}_{t, T} \otimes \ln \tilde{S}_{t} \oplus g(T) \ominus g(t)\right. \\
& \left.\quad \otimes \tilde{m}_{t, T} \oplus \tilde{\Gamma}_{t, T} \oslash \tilde{M}\right], t \in \mathcal{T},
\end{aligned}
$$

where

$$
\begin{aligned}
& \tilde{m}_{t, T}=e^{-(T-t) \otimes \tilde{\mu}}, \tilde{M} \\
& =4 \otimes \tilde{\mu}, \tilde{\xi}_{i}^{\mathbb{Q}}=\tilde{\xi}_{i} \ominus \bar{\theta}_{0}, \tilde{\lambda}^{\mathbb{Q}} \\
& =\tilde{\lambda} \otimes \bigoplus_{i=1}^{n} p_{i} \otimes \tilde{\xi}_{i} \oslash \tilde{\xi}_{i}^{\mathbb{Q}},
\end{aligned}
$$

$$
\begin{aligned}
\tilde{p}_{i}^{\mathbb{Q}}= & \left(p_{i} \otimes \tilde{\xi}_{i} \oslash \tilde{\xi}_{i}^{\mathbb{Q}}\right) \\
& \oslash\left(\bigoplus_{i=1}^{n} p_{i} \otimes \tilde{\xi}_{i} \oslash \tilde{\xi}_{i}^{\mathbb{Q}}\right), i \in\{1,2, \ldots, n\},
\end{aligned}
$$

$\tilde{\Gamma}_{t, T}=\tilde{\Gamma}_{1, t, T} \oplus \tilde{\Gamma}_{2, t, T}, \tilde{\Gamma}_{1, t, T}=\tilde{\gamma}_{1,1, t, T}$

$$
\otimes \tilde{\gamma}_{1,2, t, T},
$$

$\tilde{\gamma}_{1,1, t, T}=\tilde{\sigma} \otimes\left(1 \ominus \tilde{m}_{t, T}\right), \tilde{\gamma}_{1,2, t, T}=\tilde{\sigma}$

$$
\otimes\left(1 \oplus \tilde{m}_{t, T}\right) \oplus 4 \otimes \hat{\theta}_{0},
$$

$$
\begin{gathered}
\tilde{\Gamma}_{2, t, T}=4 \otimes \tilde{\lambda}^{\mathbb{Q}} \otimes \tilde{\gamma}_{2, t, T}, \tilde{\gamma}_{2, t, T} \\
=\bigoplus_{i=1}^{n} \tilde{\Lambda}_{i, t, T}^{\prime},
\end{gathered}
$$

$$
\begin{aligned}
& \tilde{\Lambda}_{i, t, T}^{\prime}=\tilde{p}_{i}^{\mathbb{Q}} \otimes \tilde{\Lambda}_{i, t, T}, \tilde{\Lambda}_{i, t, T} \\
& \quad=\ln \left(\left(\tilde{\xi}_{i}^{\mathbb{Q}} \ominus \tilde{m}_{t, T}\right) \oslash\left(\tilde{\xi}_{i}^{\mathbb{Q}} \ominus 1\right)\right), i \in\{1,2, \ldots, n\} .
\end{aligned}
$$

Moreover, for arbitrary $\alpha \in[0,1]$ and $\Xi \in \Sigma$

$$
\begin{aligned}
& \left(\tilde{F}^{\mathbb{Q}}(t, T)\right)_{\alpha}^{\Xi}=\exp \left[\left(\tilde{m}_{t, T}\right)_{\alpha}^{\Xi} \ln \left(\tilde{S}_{t}\right)_{\alpha}^{\Xi}\right. \\
& \quad+g(T)-\left(g(t) \bigotimes_{i n t}\left(\tilde{m}_{t, T}\right)_{\alpha}\right)^{\Xi^{\prime}} \\
& \left.\quad+\left(\left(\tilde{\Gamma}_{t, T}\right)_{\alpha} \bigotimes_{\text {int }} \tilde{M}_{\alpha}\right)^{\Xi}\right], t \in \mathcal{T},
\end{aligned}
$$

where

$$
\begin{aligned}
& \left(\tilde{m}_{t, T}\right)_{\alpha}^{\Xi}=e^{-(T-t) \tilde{\mu}_{\alpha}^{\Xi^{\prime}}}, \tilde{M}_{\alpha}^{\Xi}=4 \tilde{\mu}_{\alpha}^{\Xi},\left(\tilde{\xi}_{i}^{Q}\right)_{\alpha}^{\Xi} \\
& \quad=\left(\tilde{\xi}_{i}\right)_{\alpha}^{\Xi}-\bar{\theta}_{0},
\end{aligned}
$$




$$
\begin{aligned}
& \left(\tilde{\lambda}^{\mathbb{Q}}\right)_{\alpha}^{\Xi}=\tilde{\lambda}_{\alpha}^{\Xi} \sum_{i=1}^{n} p_{i}\left(\tilde{\xi}_{i}\right)_{\alpha}^{\Xi} /\left(\tilde{\xi}_{i}^{\mathbb{Q}}\right)_{\alpha}^{\Xi^{\prime}}, \\
& \left(\tilde{p}_{i}^{\mathbb{Q}}\right)_{\alpha}^{\Xi} \\
& =\frac{p_{i}\left(\tilde{\xi}_{i}\right)_{\alpha}^{\Xi} /\left(\tilde{\xi}_{i}^{\mathbb{Q}}\right)_{\alpha}^{\Xi^{\prime}}}{\sum_{i=1}^{n} p_{i}\left(\tilde{\xi}_{i}\right)_{\alpha}^{\Xi^{\prime}} /\left(\tilde{\xi}_{i}^{\mathbb{Q}}\right)_{\alpha}^{\Xi}}, i \in\{1,2, \ldots, n\}, \\
& \left(\tilde{\Gamma}_{t, T}\right)_{\alpha}^{\Xi} \\
& =\left(\tilde{\Gamma}_{1, t, T}\right)_{\alpha}^{\Xi}+\left(\tilde{\Gamma}_{2, t, T}\right)_{\alpha}^{\Xi},\left(\tilde{\Gamma}_{1, t, T}\right)_{\alpha}^{\Xi} \\
& =\left(\left(\tilde{\gamma}_{1,1, t, T}\right)_{\alpha} \otimes_{\text {int }}\left(\tilde{\gamma}_{1,2, t, T}\right)_{\alpha}\right)^{\Xi} \text {, } \\
& \left(\tilde{\gamma}_{1,1, t, T}\right)_{\alpha}^{\Xi} \\
& =\tilde{\sigma}_{\alpha}^{\Xi}\left(1-\left(\tilde{m}_{t, T}\right)_{\alpha}^{\Xi^{\prime}}\right),\left(\tilde{\gamma}_{1,2, t, T}\right)_{\alpha}^{\Xi} \\
& =(\tilde{\sigma})_{\alpha}^{\Xi}\left(1+\left(\tilde{m}_{t, T}\right)_{\alpha}^{\Xi}\right)+4 \hat{\theta}_{0} \text {, } \\
& \left(\tilde{\Gamma}_{2, t, T}\right)_{\alpha}^{\Xi} \\
& =4\left(\left(\tilde{\lambda}^{\mathbb{Q}}\right)_{\alpha} \otimes_{i n t}\left(\tilde{\gamma}_{2, t, T}\right)_{\alpha}\right)^{\Xi},\left(\tilde{\gamma}_{2, t, T}\right)_{\alpha}^{\Xi} \\
& =\sum_{i=1}^{n}\left(\tilde{\Lambda}_{i, t, T}^{\prime}\right)_{\alpha}^{\Xi} \text {, } \\
& \left(\tilde{\Lambda}_{i, t, T}^{\prime}\right)_{\alpha}^{\Xi} \\
& =\left(\left(\tilde{p}_{i}^{\mathbb{Q}}\right)_{\alpha} \otimes_{i n t}\left(\tilde{\Lambda}_{i, t, T}\right)_{\alpha}\right)^{\Xi},\left(\tilde{\Lambda}_{i, t, T}\right)_{\alpha}^{\Xi} \\
& =\ln \frac{\left(\tilde{\xi}_{i}^{\mathbb{Q}}\right)_{\alpha}^{\Xi}-\left(\tilde{m}_{t, T}\right)_{\alpha}^{\Xi^{\prime}}}{\left(\tilde{\xi}_{i}^{\mathbb{Q}}\right)_{\alpha}^{\Xi^{\prime}}-1}, \\
& i \in\{1,2, \ldots, n\} \text {. }
\end{aligned}
$$

Proof Formula (7) can be written in the following form:

$$
\begin{aligned}
& F^{\mathbb{Q}}(t, T)=\exp \left[m_{t, T} \ln S_{t}+g(T)-m_{t, T} g(t)\right. \\
& \left.\quad+\frac{\sigma\left(1-m_{t, T}\right)\left(\sigma\left(1+m_{t, T}\right)+4 \hat{\theta}_{0}\right)+4 \lambda^{\mathbb{Q}} \sum_{i=1}^{n} p_{i}^{\mathbb{Q}} \ln \frac{\xi_{i}^{\mathbb{Q}}-m_{t, T}}{\xi_{i}^{\mathbb{Q}}-1}}{4 \mu}\right],
\end{aligned}
$$

w h e r e $\quad m_{t, T}=e^{-\mu(T-t)}, \quad \xi_{i}^{\mathbb{Q}}=\xi_{i}-\bar{\theta}_{0}$, $p_{i}^{\mathbb{Q}}=\frac{p_{i} \frac{\xi_{i}}{\xi_{i}^{\mathbb{Q}}}}{\sum_{i=1}^{n} p_{\frac{\xi_{i}}{\xi_{i}}}}, i \in\{1,2, \ldots, n\}$, and $\lambda^{\mathbb{Q}}=\lambda \sum_{i=1}^{n} p_{i} \frac{\xi_{i}}{\xi_{i}^{\mathbb{Q}}}$. Therefore (22-28) hold.

Functions $\exp (x)$ and $\ln (x)$ are increasing. Additionally they fulfill the assumptions of Proposition 2.3 from $\mathrm{Wu}$ (2004). Thus, for arbitrary fuzzy number $\tilde{a}$
$\left(e^{\tilde{a}}\right)_{\alpha}=\left[e^{\tilde{a}_{\alpha}^{L}}, e^{\tilde{a}_{\alpha}^{U}}\right]$

and for arbitrary positive fuzzy number $\tilde{b}$

$(\ln \tilde{b})_{\alpha}=\left[\ln \tilde{b}_{\alpha}^{L}, \ln \tilde{b}_{\alpha}^{U}\right]$.

Since $\tilde{\mu}$ and $\tilde{\xi}_{i} \ominus \bar{\theta}_{0} \ominus 1, i \in\{1,2, \ldots, n\}$, are positive, $1 \ominus \tilde{m}_{t, T}$ and $\tilde{\xi}_{i}^{\mathbb{Q}} \ominus \tilde{m}_{t, T}, i \in\{1,2, \ldots, n\}$, are also positive fuzzy numbers. By straightforward computations, applying (37) and (38), we obtain

$$
\begin{aligned}
& \left(\tilde{m}_{t, T}\right)_{\alpha}^{\Xi} \\
& =e^{-(T-t) \tilde{\mu}_{\alpha}^{\Xi^{\prime}}}, \tilde{M}_{\alpha}^{\Xi} \\
& =4 \tilde{\mu}_{\alpha}^{\Xi},\left(\tilde{\xi}_{i}^{\mathbb{Q}}\right)_{\alpha}^{\Xi}=\left(\tilde{\xi}_{i}\right)_{\alpha}^{\Xi}-\bar{\theta}_{0}, \\
& \left(\tilde{p}_{i}^{\mathbb{Q}}\right)_{\alpha}^{\Xi} \\
& =\frac{\left(p_{i} \otimes \tilde{\xi}_{i} \oslash \tilde{\xi}_{i}^{\mathbb{Q}}\right)_{\alpha}^{\Xi}}{\left(\bigoplus_{i=1}^{n} p_{i} \otimes \tilde{\xi}_{i} \oslash \tilde{\xi}_{i}^{\mathbb{Q}}\right)_{\alpha}^{\Xi^{\prime}}} \\
& =\frac{p_{i}\left(\tilde{\xi}_{i}\right)_{\alpha}^{\Xi} /\left(\tilde{\xi}_{i}^{\mathbb{Q}}\right)_{\alpha}^{\Xi^{\prime}}}{\sum_{i=1}^{n} p_{i}\left(\tilde{\xi}_{i}\right)_{\alpha}^{\Xi^{\prime}} /\left(\tilde{\xi}_{i}^{\mathbb{Q}}\right)_{\alpha}^{\Xi}, i \in\{1,2, \ldots, n\},} \\
& \left(\tilde{\lambda}^{\mathbb{Q}}\right)_{\alpha}^{\Xi} \\
& =\tilde{\lambda}_{\alpha}^{\Xi}\left(\bigoplus_{i=1}^{n} p_{i} \otimes \tilde{\xi}_{i} \oslash \tilde{\xi}_{i}^{\mathbb{Q}}\right)_{\alpha}^{\Xi} \\
& =\tilde{\lambda}_{\alpha}^{\Xi} \sum_{i=1}^{n} p_{i}\left(\tilde{\xi}_{i}\right)_{\alpha}^{\Xi} /\left(\tilde{\xi}_{i}^{\mathbb{Q}}\right)_{\alpha}^{\Xi^{\prime}},
\end{aligned}
$$

and

$$
\begin{aligned}
\left(\tilde{F}^{\mathbb{Q}}(t, T)\right)_{\alpha}^{\Xi} & \\
= & \exp \left[\tilde{m}_{t, T} \otimes \ln \tilde{S}_{t} \oplus g(T) \ominus g(t) \otimes \tilde{m}_{t, T} \oplus \tilde{\Gamma}_{t, T}\right. \\
& \oslash(4 \otimes \tilde{\mu})]_{\alpha}^{\Xi} \\
= & \exp \left[\left(\tilde{m}_{t, T} \otimes \ln \tilde{S}_{t}\right)_{\alpha}^{\Xi}+g(T)\right. \\
& \left.-\left(g(t) \otimes \tilde{m}_{t, T}\right)_{\alpha}^{\Xi^{\prime}}+\left(\tilde{\Gamma}_{t, T} \oslash \tilde{M}\right)_{\alpha}^{\Xi}\right] \\
= & \exp \left[\left(\tilde{m}_{t, T}\right)_{\alpha}^{\Xi} \ln \left(\tilde{S}_{t}\right)_{\alpha}^{\Xi}+g(T)-(g(t)\right. \\
& \left.\left.\bigotimes_{i n t}\left(\tilde{m}_{t, T}\right)_{\alpha}\right)^{\Xi^{\prime}}+\left(\left(\tilde{\Gamma}_{t, T}\right)_{\alpha} \oslash_{i n t} \tilde{M}_{\alpha}\right)^{\Xi}\right] .
\end{aligned}
$$

Thus, formulas (29-32) hold. In a similar way, we obtain (33-35). Finally, equality (38) implies (36), which finishes the proof.

Fuzzy-valued functions and constants $\tilde{m}_{t, T}, \tilde{\Gamma}_{t, T}, \tilde{\Gamma}_{1, t, T}, \tilde{\Gamma}_{2, t, T}$, $\tilde{\gamma}_{1,1, t, T}, \tilde{\gamma}_{1,2, t, T}, \tilde{M}, \tilde{\lambda}^{\mathbb{Q}}, \tilde{\xi}_{i}^{\mathbb{Q}}, \tilde{p}_{i}^{\mathbb{Q}}, \tilde{\Lambda}_{i, t, T}^{\prime}, \tilde{\Lambda}_{i, t, T}$ for $i \in\{1,2, \ldots, n\}$, as well as their $\alpha$-level sets, introduced in the above theorem, 
are auxiliary. They are used to simplify the fuzzy forward price formula.

A straightforward consequence of Theorem 2 is the following corollary.

Corollary 1 The fuzzy forward price $\tilde{K}_{t}$ of a forward contract calculated at time $t$ is given by the following formula:

$\tilde{K}_{t}=\frac{1}{N} \otimes \bigoplus_{i=1}^{N} \tilde{F}^{\mathbb{Q}}\left(t, T_{i}\right)$.

Moreover, for arbitrary $\alpha \in[0,1]$ and $\Xi \in \Sigma$

$\left(\tilde{K}_{t}\right)_{\alpha}^{\Xi}=\frac{1}{N} \sum_{i=1}^{N}\left(\tilde{F}^{\mathbb{Q}}\left(t, T_{i}\right)\right)_{\alpha}^{\Xi}$.

\section{Decision making in fuzzy environment}

We apply a modified version of a method of investment decision-making, which was used, i.e. by Nowak and Pawłowski $(2017,2019)$ and the first time by Piasecki (2014) in another context.

We assume that $t \in[0, T]$. Let $\hat{K}_{t}$ be the market price of the considered forward price of a forward contract calculated at time $t$. We denote by $V=\{\mathbf{B}, \mathbf{H}, \mathbf{S}\}$ the set of investment decisions: B (to buy; when the contract is undervalued), $\mathbf{H}$ (to hold; when the contract is fairly valued), and $\mathbf{S}$ (to sell; when the contract is overvalued). The advice choice function $\Lambda: \mathbb{R}^{2} \rightarrow 2^{V}$ has the form:

$\mathbf{B} \in \Lambda\left(K_{t}, \hat{K}_{t}\right) \Leftrightarrow \hat{K}_{t}<K_{t} ;$

$\mathbf{H} \in \Lambda\left(K_{t}, \hat{K}_{t}\right) \Leftrightarrow \hat{K}_{t}=K_{t}$

$\mathbf{S} \in \Lambda\left(K_{t}, \hat{K}_{t}\right) \Leftrightarrow \hat{K}_{t}>K_{t}$.

From the Zadeh Extension Principle, one can obtain the extended advice choice function $\tilde{\Lambda}:[0,1]^{\mathbb{R}} \times \mathbb{R} \rightarrow[0,1]^{V}$. If the symbol $\tilde{l}$ denotes the membership function of $\tilde{\Lambda}\left(\tilde{K}_{t}, \hat{K}_{t}\right)$, then

$$
\begin{aligned}
\tilde{l}(\mathbf{B}) & =\min \left(\delta_{\tilde{K}_{t}}\left(\hat{K}_{t}\right),\left(1-\beta_{\tilde{K}_{t}}\left(\hat{K}_{t}\right)\right)\right) ; \\
\tilde{l}(\mathbf{H}) & =\min \left(\delta_{\tilde{K}_{t}}\left(\hat{K}_{t}\right), \beta_{\tilde{K}_{t}}\left(\hat{K}_{t}\right)\right) ; \\
\tilde{l}(\mathbf{S}) & =\min \left(\beta_{\tilde{K}_{t}}\left(\hat{K}_{t}\right),\left(1-\delta_{\tilde{K}_{t}}\left(\hat{K}_{t}\right)\right)\right),
\end{aligned}
$$

where for $\hat{x} \in \mathbb{R}$ :

$$
\begin{gathered}
\beta_{\tilde{K}_{t}}(\hat{x})=\sup \left\{\mu_{\tilde{K}_{t}}(x): x \leq \hat{x}\right\} \\
=\left\{\begin{array}{cc}
\mu_{\tilde{K}_{t}}(\hat{x}) \text { for }\left(\tilde{K}_{t}\right)_{0}^{L} \leq \hat{x} \leq\left(\tilde{K}_{t}\right)_{1}^{L} ; \\
\text { otherwise }
\end{array}\right. \\
\delta_{\tilde{K}_{t}}(\hat{x})=\sup \left\{\mu_{\tilde{K}_{t}}(x): x \geq \hat{x}\right\} \\
=\left\{\begin{array}{cc}
\mu_{\tilde{K}_{t}}(\hat{x}) \text { for }\left(\tilde{K}_{t}\right)_{1}^{U} \leq \hat{x} \leq\left(\tilde{K}_{t}\right)_{0}^{U} ; \\
1 & \text { otherwise. }
\end{array}\right.
\end{gathered}
$$

To compute values of the membership function $\mu_{\tilde{K}}$, one can use the bisection search (see, e.g. Wu 2004). The obtained $\alpha$ -level set $\tilde{\Lambda}\left(\tilde{K}_{t}, \hat{K}_{t}\right)_{\alpha}$ contains recommendations for a financial analyst.

\section{Numerical examples}

According to Theorem 1, one may compute the forward price in a closed-form. It allows to calibrate the model to the observed on the market prices of forward contracts by disambiguating values of the market price of diffusion and jump risks, i.e. uniquely determine the risk-neutral measure $\mathbb{Q}$. Thanks to pinning down the risk neutral measure out of uncountable many possibilities, the model is suited to how the market players perceive the future levels of prices at the moment of calibration. Afterwards, one obtains the form of the whole forward curve and therefore valuation of nonstandardized, over-the-counter contracts, is feasible.

Traditional, non-fuzzy models require point estimation of their parameters. It is widely known that on exceptionally volatile energy markets values of observed, implied or hidden parameters may rapidly change, they are themselves extremely volatile. Their estimators based on historical data are dependent on the range of data selected to the analysis. What is more, the market implied (e.g. from exchange price quotations of liquid forward contracts or options) parameters are correct only for some short period of time, inasmuch as energy markets evolve dynamically. Moreover, point estimation by its nature may be encumbered with errors. Therefore, it is often more adequate to rely on some set of values rather than on a single estimator.

This is why our approach goes one step further. "Pricing forward contracts with fuzzy parameters" illustrates how to introduce fuzziness into the model. We assume that the parameters described therein are triangular fuzzy numbers which may be obtained from experts or market practitioners who indicate the smallest, greatest and most probable values of the parameters (which uniquely constitute triangular fuzzy numbers). Experts, not infrequently, are guided by statistical methods to set the modal values and ends of the fuzzy numbers.

Theorem 2 with Corollary 1 outline the forms of the fuzzy forward prices of forward contracts and their $\alpha$-level 
sets. The following section exemplifies different properties of the fuzzy forward prices on real-life trading cases.

\subsection{Price's $\alpha$-level sets, membership function, sensitivity analysis}

Let us assume that the crisp parameters, modal values and ends of the triangular fuzzy numbers have been estimated by experts from some historical data (see Table 1).

For $t=0, g_{t}=4.6, p_{1}=0.4, p_{2}=0.6$ the market price of diffusion risk $\hat{\theta}_{0}$ and the market price of jump risk $\bar{\theta}_{0}$ have been calibrated to the set of observed on the market liquid forward contracts with quoted prices 114, 116, $112,116.75$ (on the exchange one can see only forward prices of the respective forward contracts, cf. Definition 3 ) and respective values of the seasonality parameter $g_{T}$ equal to $4.75,4.73,4.74,4.76$, corresponding to the three monthly contracts with delivery of electricity during $T \in\left[0, \frac{1}{12}\right], T \in\left[\frac{1}{12}, \frac{2}{12}\right], T \in\left[\frac{2}{12}, \frac{3}{12}\right]$ and one quarterly with $T \in\left[\frac{3}{12}, \frac{6}{12}\right]$, by minimizing the mean square

Table 1 Fuzzy parameters chosen for the fuzzy forward contracts' price analysis

\begin{tabular}{ll}
\hline$\tilde{S}$ & $(95,100,105)$ \\
$\tilde{\mu}$ & $(110,115,120)$ \\
$\tilde{\sigma}$ & $(1.9,2.2,2.5)$ \\
$\tilde{\lambda}$ & $(20,22.5,25)$ \\
$\tilde{\xi_{1}}$ & $(10,10.5,11)$ \\
$\tilde{\xi}_{2}$ & $(14,14.5,15)$ \\
\hline
\end{tabular}

error calculated for the analytical crisp forward prices of forward contracts (cf. Theorem 1 and formula (39)) and for the observed on the market prices of liquid forward contracts. As a result, $\hat{\theta}_{0}=-0.95, \bar{\theta}_{0}=-10.45$.

Having calibrated the model, let us assume that we want to price the non-standardized forward contract with $T \in\left[\frac{1}{48}, \frac{1}{24}\right]$. The relationship between the $\alpha$-level sets of the fuzzy price of the forward contract and the value of $\alpha$ is shown in Fig. 1. The crisp value of the forward contract is 111.92 for $g_{T}=4.72$.

Figure 2 presents the membership function $\mu_{\tilde{K}_{0}}$ of the fuzzy forward price of the forward contract with the aforementioned parameters.

One may draw interesting conclusions from the sensitivity analysis of 0.9-level sets of the fuzzy forward contract's price with respect to the fuzzy speed of mean reversion of the price, i.e. $\tilde{\mu}$. As shown in Fig. 3, the bigger the modal values of $\tilde{\mu}$ (the length of the support does not change), the narrower the supports of the 0.9-level sets of the fuzzy forward contract's price, whereas the level of its modal values does not change. Intuitively, with the increasing pace of mean reversion, the underlying asset's price is less volatile (more "centered" around its seasonality) which results in more stable valuation of the fuzzy forward contract's price.

Another valuable illustration of the model properties is an analogous sensitivity analysis, but with respect to the intensity $\tilde{\lambda}$ of the jump process, see Fig. 4 . One may note that inasmuch as we allow positive jumps only, increasing the modal value of the lambda fuzzy parameter (with values of

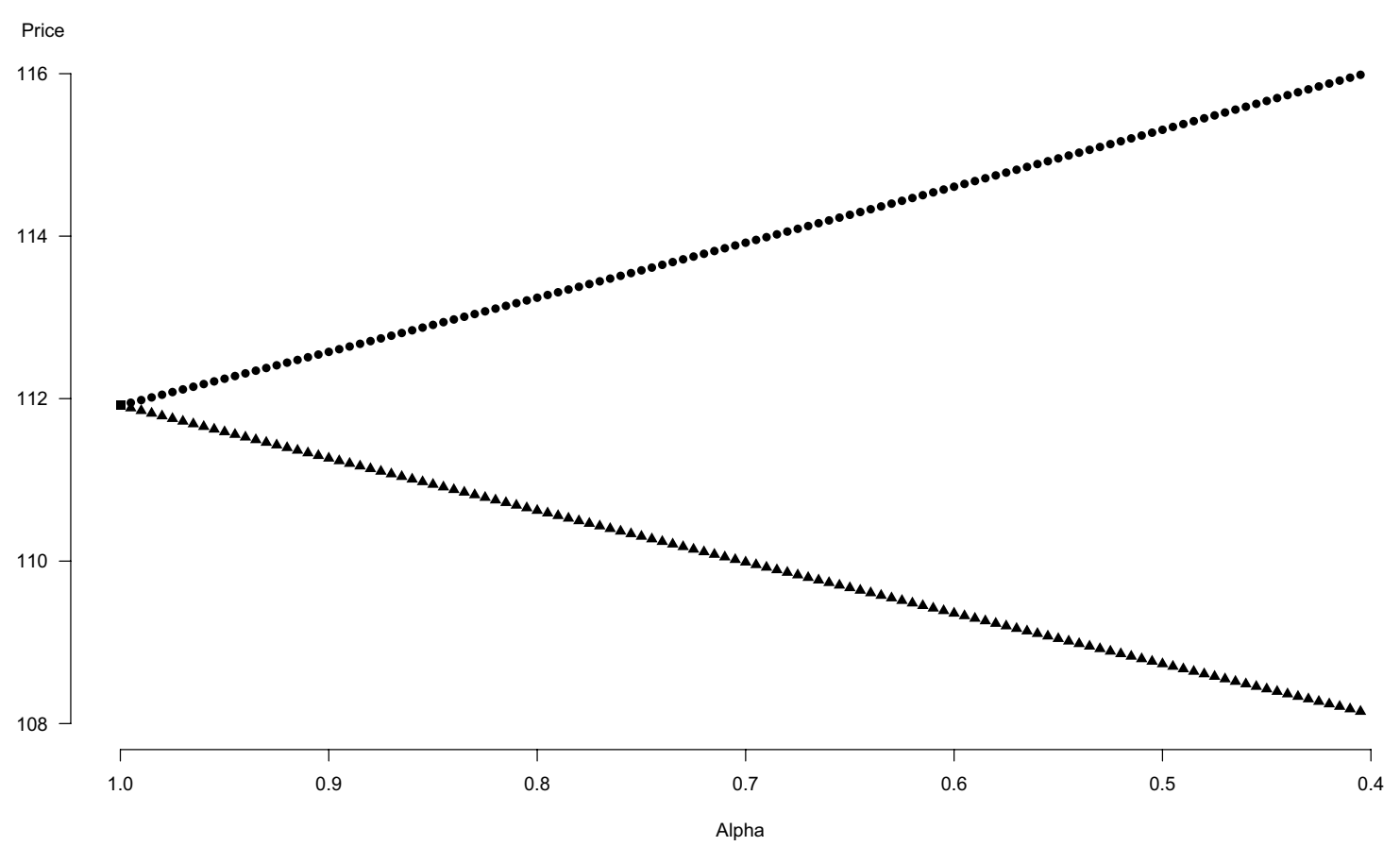

Fig. $1 \alpha$-level sets' ends (circles: right ends, triangles: left ends) of the forward contract's price depending on the membership degree $\alpha$ 
Membership degree

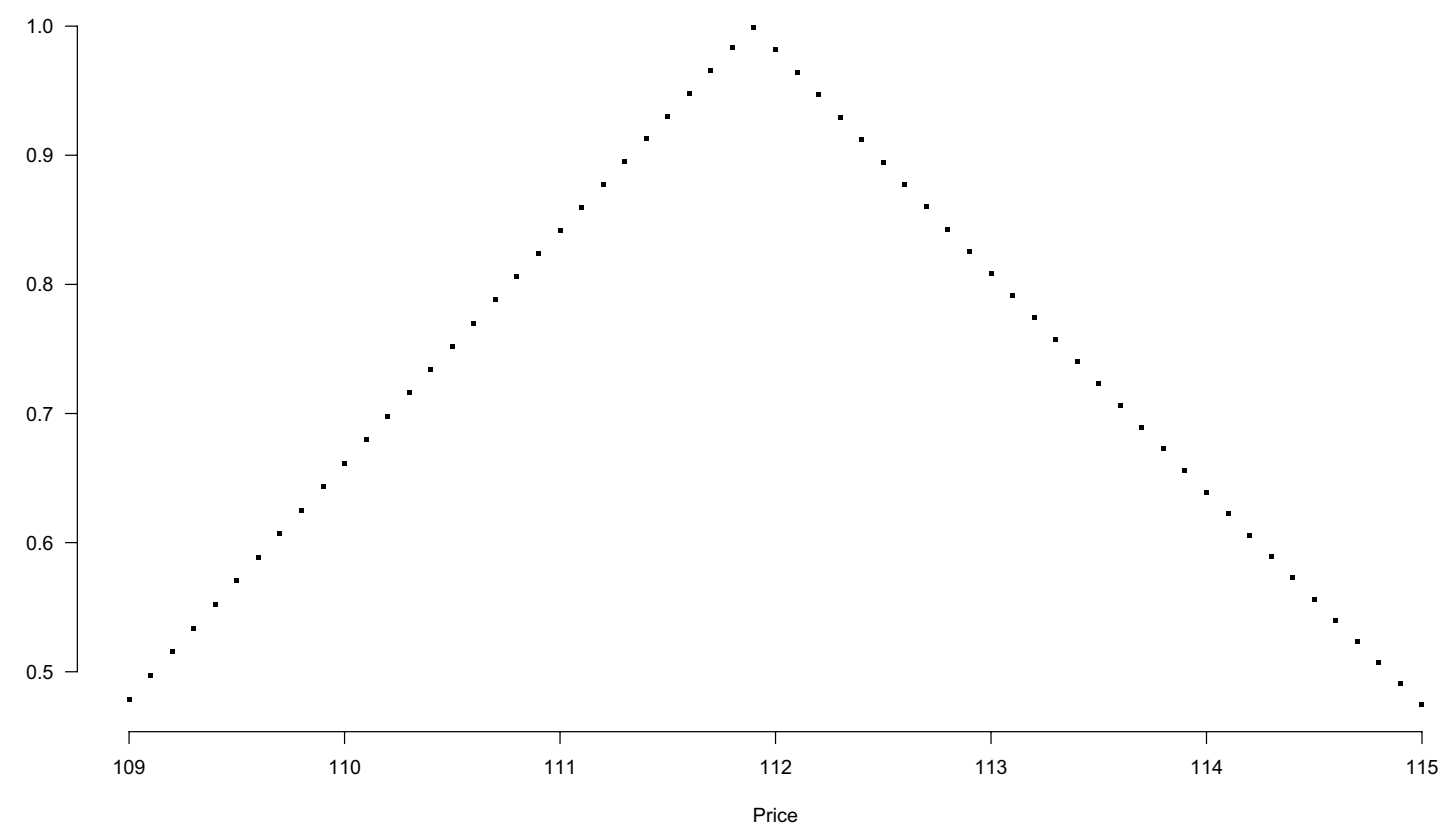

Fig. 2 The membership function of the fuzzy forward contract's price

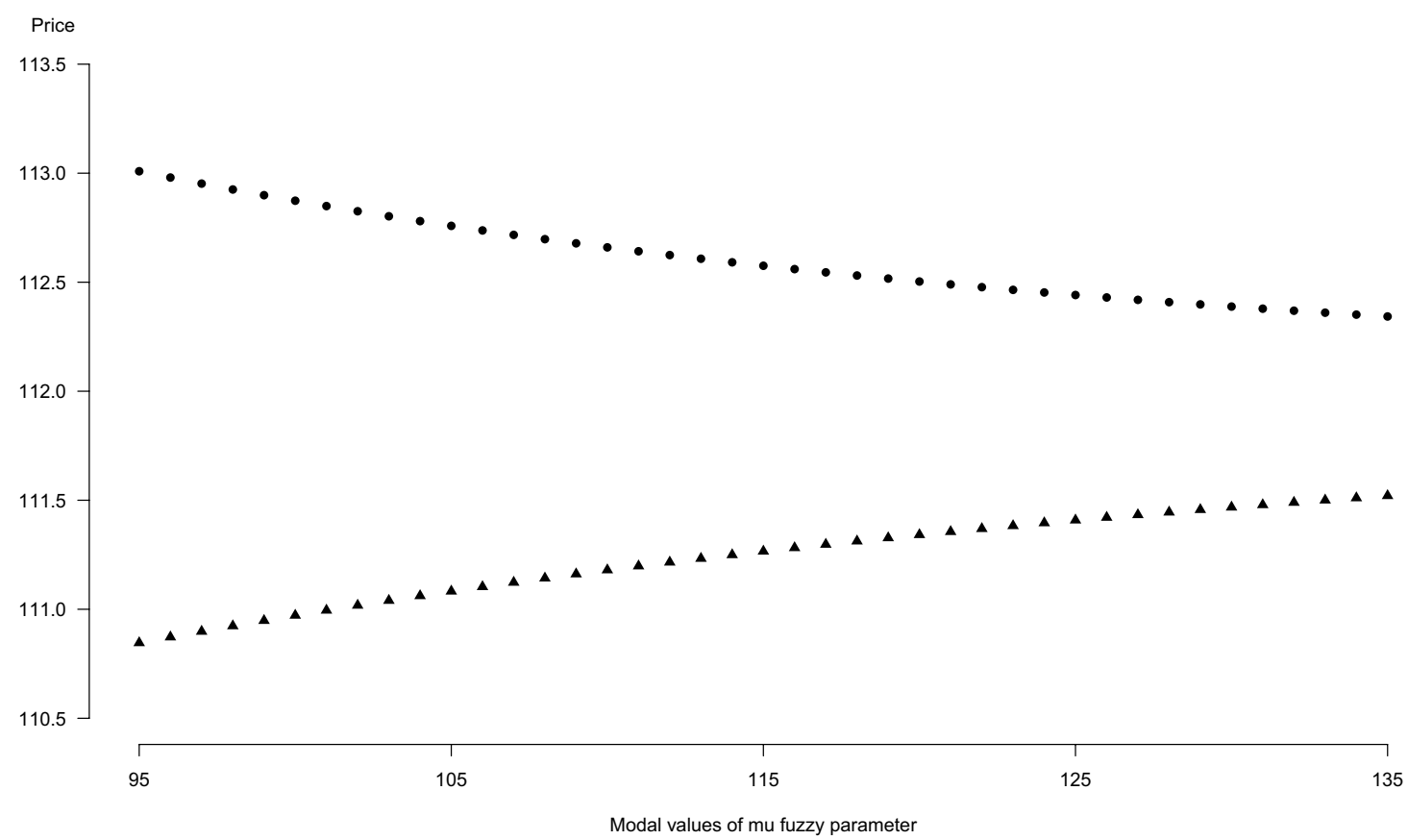

Fig. 3 Sensitivity analysis of 0.9-level sets of the fuzzy forward contract's price to changing triangular number of the parameter $\mu$ (circles: right ends of the prices' intervals, triangles: left ends of the prices' intervals)

other parameters unchanged)—making big jumps of the spot price occur more frequently - the fuzzy forward contract's price increases as well.

\subsection{Forward curve in the fuzzy environment}

Following the calibration of the model to the prices of liquid forward contracts quoted on the exchange presented 


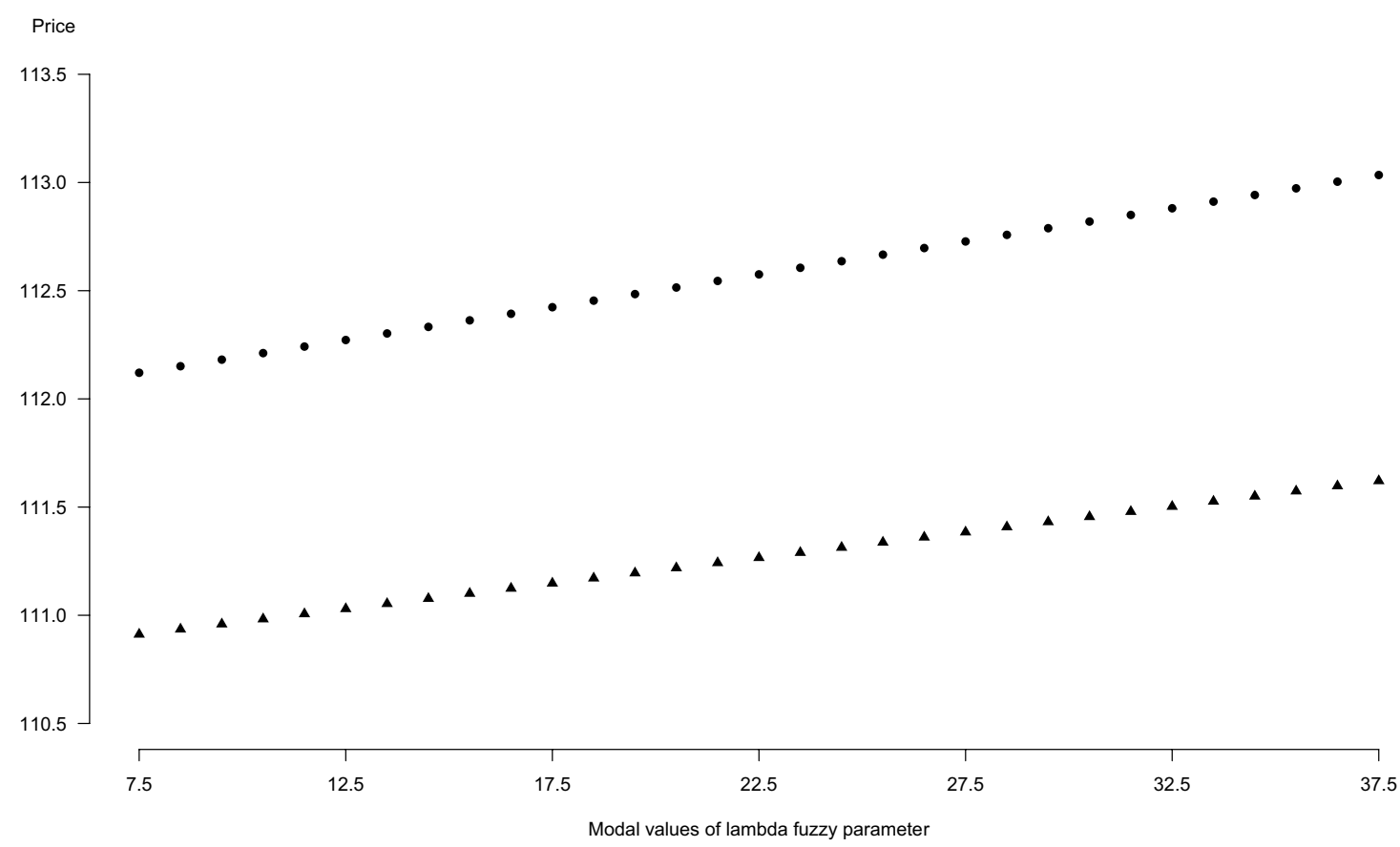

Fig. 4 Sensitivity analysis of 0.9-level sets of the fuzzy forward contract's price to changing triangular number of the parameter $\lambda$ (circles: right ends of the prices' intervals, triangles: left ends of the prices' intervals)

in "Price's $\alpha$-level sets, membership function, sensitivity analysis" and assuming the same set of values of the fuzzy parameters (see Table 1) we price four consecutive weekly and one two-week non-standardized forward contracts with respective values of the seasonality parameter $g_{T}: 4.73,4.72,4.77,4.75$ and 4.74 by using formulas (39) and (40). In Fig. 5 one can observe the beginning of

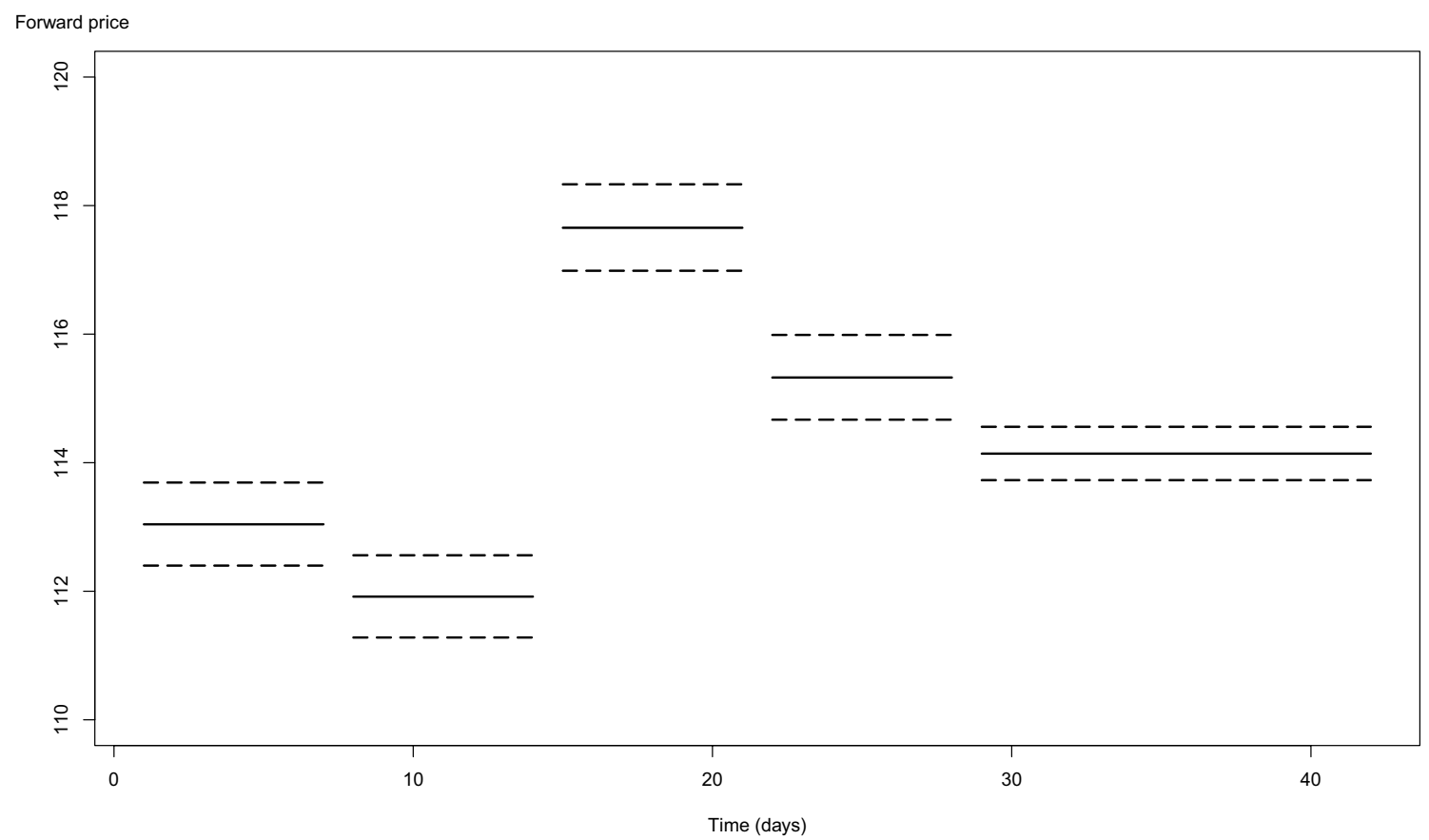

Fig. 5 Fuzzy forward contracts' prices (solid line: crisp valuation, dashed line: left and right ends of the fuzzy prices' 0.9-level sets ends) 
the forward curve (as described above) obtained by crisp valuation enriched with ends of 0.9-level sets ends.

\subsection{Application of the method-increased jump intensity}

Let us discuss the real-life application of the fuzzy pricing on electricity market. Assume that we valuate the non-standardized forward contract described in "Price's $\alpha$-level sets, membership function, sensitivity analysis" which has a crisp price equal to 111.92 and that the market participants start to anticipate a period of increased frequency of jumps (encapsulating the energy delivery period for $\left.T \in\left[\frac{1}{48}, \frac{1}{24}\right]\right)$ in spot electricity prices being the result of high probability of a drought causing turning off power blocks cooled by water from rivers. Nevertheless, the modal values of the fuzzified model parameters have been statistically estimated (e.g., from historical data) and the market prices of risk have been calibrated to the last observed, quoted prices of liquid forward contracts, cf. "Price's $\alpha$-level sets, membership function, sensitivity analysis". In such a situation, after consulting an expert, the right end of the fuzzy intensity parameter $\tilde{\lambda}$ may be adequately shifted to reflect the maximum predicted number of spot price spikes within the period of delivery of energy (we can assume, e.g., ten times increased intensity), simultaneously leaving the left end and the modal value of the fuzzy number unchanged (reflecting the base, i.e., without outages, scenario). Then, $\tilde{\lambda}=(20,22.5,250)$ and the 0.85-level set of the fuzzy price of this forward contract is equal to $[110.97,113.8]$, where the other fuzzy parameters are set as in Table 1 . By contrast, for $\tilde{\lambda}=(20$, $22.5,25)$ the 0.85 -level set is equal to $[110.97,112.88]$. The value 113.8 indicates the reasonable forward price which may be observed on the market prior to delivery, if the extreme frequency of spikes in the spot prices scenario assumed by analysts comes true.

\subsection{Automatized investment decision-making}

Let us assume the same set of the fuzzy parameters as in Table 1 and consider the situation in which the market price of the forward contract with delivery period $T \in\left[\frac{3}{12}, \frac{4}{12}\right]$ differs from the modal value of the fuzzy forward price of this contract equal to 115.26 for $g_{T}=4.75$ (which is usually the case because the fuzzy parameters are expert based while the market prices evolve continuously). An investor fixes $\alpha$ at 0.9 level, so that he selects recommendations from the 0.9-level set $\tilde{\Lambda}\left(\tilde{K}_{t}, \hat{K}_{t}\right)_{0.9}$ which depend on the actual market forward contract price $\hat{K}_{t}$ - see Table 2 with three exemplary market price situations and resulting investment decisions.
Table 2 Automatized decision-making for three market forward contract prices' scenarios and the modal value of the fuzzy forward price equal to 115.26

\begin{tabular}{llll}
\hline$\hat{K}_{t}$ & $\tilde{l}(\mathbf{B})$ & $\tilde{l}(\mathbf{H})$ & $\tilde{l}(\mathbf{S})$ \\
\hline 112.5 recommendations & 1 & 0 & 0 \\
& B: yes & H: no & S: no \\
115 recommendations & 0.091 & 0.909 & 0 \\
& B: no & H: yes & S: no \\
118.25 recommendations & 0 & 0.048 & 0.952 \\
& B: no & H: no & S: yes \\
\hline
\end{tabular}

\section{Conclusions}

In the paper, the authors have introduced the new model for the electricity spot, taking into account all the specificity of the electrical energy prices. To this end, we have proposed the mean-reverting jump-diffusion process of the deseasonalised logarithms of electricity prices with the hyperexponential jump-size distribution.

The analytical formulas for the forward contracts' prices have been derived both in crisp and fuzzy cases. In the crisp case, the derivation required applying an advanced probabilistic method based on Esscher transformation for change of probability measure. At this stage, identifying the form of the price process with respect to the risk-neutral probability measure plays a crucial role. The obtained analytical formula incorporates the market prices of diffusion and jump risks. In turn, in the fuzzy case, the derivation of the pricing formulas involved the usage of elements of fuzzy sets theory.

The theoretical results have been followed by numerical illustrations of selected properties of the fuzzy prices. Triangular shapes of both the membership function and $\alpha$ -level sets' ends of the fuzzy forward prices (as a function of $\alpha$ ), calculated and presented for the sample configuration of the model's parameters, are preserved (as compared to the triangular fuzzy model's parameters), acknowledging the stability of the method, cf. "Price's $\alpha$-level sets, membership function, sensitivity analysis". Similarly, outcomes of the performed sensitivity analysis of the $\alpha$-level sets of the fuzzy forward contract's price with respect to the selected model's parameters are concordant with the anticipated model's behaviour.

"Forward curve in the fuzzy environment" has given an image of what the forward curve might look like in a fuzzy environment.

"Application of the method-increased jump intensity" has been provided to exemplify one of the possible real-life applications of the fuzzy pricing and the influence of incertitude about some model's parameter on the value of the fuzzy price of a forward contract.

The investment decision-making procedure for forward contracts based on the relation between their theoretical 
fuzzy valuation and market quotes has been presented on real-life examples, cf. "Automatized investment decision-making".

Possible future work directions include valuation of options on delivery of electricity and creation of a new multi-factor model of dynamics of deseasonalised logarithms of electricity prices with stochastic volatility in the fuzzy environment.

Data availability All data generated or analysed during this study are included in this published article.

Open Access This article is licensed under a Creative Commons Attribution 4.0 International License, which permits use, sharing, adaptation, distribution and reproduction in any medium or format, as long as you give appropriate credit to the original author(s) and the source, provide a link to the Creative Commons licence, and indicate if changes were made. The images or other third party material in this article are included in the article's Creative Commons licence, unless indicated otherwise in a credit line to the material. If material is not included in the article's Creative Commons licence and your intended use is not permitted by statutory regulation or exceeds the permitted use, you will need to obtain permission directly from the copyright holder. To view a copy of this licence, visit http://creativecommons.org/licenses/by/4.0/.

\section{References}

Anzilli L, Facchinetti G, Pirotti T (2018) Pricing of minimum guarantees in life insurance contracts with fuzzy volatility. Info Sci 460-461:578-593

Bao J, Zhao Y (2019) Option pricing in Markov-modulated exponential Lévy models with stochastic interest rates. J Comput Appl Math 357:146-160

Barndorff-Nielsen OE (1998) Processes of normal inverse gaussian type. Finance Stoch 2(1):41-68

Benth FE, Saltyte-Benth J (2004) The normal inverse Gaussian distribution and spot price modeling in energy markets. Int $\mathrm{J}$ Theor Appl Finance 7(2):177-192

Benth FE, Sgarra C (2012) The risk premium and the Esscher transform in power markets. Stoch Anal Appl 30(1):20-43

Benth FE, Kiesel R, Nazarova A (2011) A critical empirical study of three electricity price models. Rev Deriv Res 34(5):1589-1616

Biancardi M, Villani G (2017) A fuzzy approach for R\&D compound option valuation. Fuzzy Sets Syst 310:108-121

Buckley J, Eslami E (2007) Pricing stock options using fuzzy sets. Iran J Fuzzy Syst 4(2):1-14

Cai N, Kou SG (2011) Option pricing under a mixed-exponential jump diffusion model. Manage Sci 57(11):2067-2081

Canakoglu E, Adiyeke E (2020) Comparison of electricity spot price modelling and risk management applications. Energies 13(18):4698

Cartea A, Figueroa M (2005) Pricing in electricity markets: a mean reverting jump diffusion model with seasonality. Appl Math Finance 12(4):313-335

Chrysafis KA, Papadopoulos BK (2009) On theoretical pricing of options with fuzzy estimators. J Comput Appl Math 223(2):552-566
Dash JK, Panda S, Panda GB (2021) A new method to solve fuzzy stochastic finance problem. J Econ Stud. https://doi.org/10. 1108/JES-10-2020-0521

de Andres-Sanchez J (2018) Pricing European options with triangular fuzzy parameters: assessing alternative triangular approximations in the Spanish stock option market. Int J Fuzzy Syst 20:1624-1643

de Jong C, Huisman R (2002) Option formulas for mean-reverting power prices with spikes. Energy Global Research Paper

Deelstra G, Simon M (2017) Multivariate European option pricing in a Markov-modulated Lévy framework. J Comput Appl Math 317:171-187

Feldmann A, Whitt W (1998) Fitting mixtures of exponentials to long-tail distributions to analyze network performance models. Perform Eval 31(3-4):245-279

Feng C, Tan J, Jiang Z, Chen S (2020) A generalized European option pricing model with risk management. Phys A: Stat Mech Appl 545:123797

Geman H, Roncoroni A (2006) Understanding the fine structure of electricity prices. J Bus 79(3):1225-1261

Gil-Lafuente A (2005) Fuzzy logic in financial analysis. Springer, Berlin

Janczura J, Weron R (2010) An empirical comparison of alternate regime-switching models for electricity spot prices. Energy Econ 32(5):1059-1073

Li H, Ware A, Di L, Yuan G, Swishchuk A, Yuan S (2018) The application of nonlinear fuzzy parameters PDE method in pricing and hedging European options. Fuzzy Sets Syst 331:14-25

Lindstrom E, Regland F (2012) Modelling extreme dependence between European electricity markets. Energy Econ 34(4):899-904

Liu WQ, Li SH (2013) European option pricing model in a stochastic and fuzzy environment. Appl Math 28(3):321-334

Lucia J, Schwartz E (2002) Electricity prices and power derivatives: evidence from the Nordic power exchange. Rev Deriv Res 5(1):5-50

Madan DB, Seneta E (1990) The variance gamma (v.g.) model for share market returns. J Bus 63(4):511-524

Muzzioli S, Torricelli C (2004) A multiperiod binomial model for pricing options in a vague world. J Econ Dyn Control 28(5):861-887

Nomikos N, Soldatos OA (2010) Analysis of model implied volatility for jump diffusion models: empirical evidence from the Nordpool market. Energy Econ 32(2):302-312

Nowak P (2002) On Jacod-Grigelionis characteristics for Hilbert space valued semimartingales. Stoch Anal Appl 20(5):963-998

Nowak P (2011) Option pricing with Levy process in a fuzzy framework. In: Atanassov K, Homenda W, Hryniewicz O, Kacprzyk J, Krawczak M, Nahorski Z, Szmidt E, Zadrozny S (eds), Recent advances in fuzzy sets, intuitionistic fuzzy sets, generalized nets and related topics. Polish Academy of Sciences

Nowak P, Pawłowski M (2017) Option pricing with application of Levy processes and the minimal variance equivalent Martingale measure under uncertainty. IEEE Trans Fuzzy Syst 25(2):402-416

Nowak P, Pawłowski M (2019) Pricing European options under uncertainty with application of Levy processes and the minimal $L^{q}$ equivalent martingale measure. J Comput Appl Math 345:416-433

Nowak P, Romaniuk M (2010) Computing option price for Levy process with fuzzy parameters. Eur J Operat Res 201(1):206-210

Nowak P, Romaniuk M (2013a) Application of the one-factor affine interest rate models to catastrophe bonds pricing. Research Report SRI PAS

Nowak P, Romaniuk M (2013b) A fuzzy approach to option pricing in a Levy process setting. Int J Appl Math Comput Sci 23(3):613-622 
Nowak P, Romaniuk M (2014) Application of Levy processes and Esscher transformed martingale measures for option pricing in fuzzy framework. J Comput Appl Math 263:129-151

Piasecki K (2014) On imprecise investment recommendations. Stud Log Gramm Rhetor 37(1):179-194

Puri ML, Ralescu DA (1986) Fuzzy random variables. J Math Anal Appl 114(2):409-422

Qin X, Lin X-W, Shang Q (2020) Fuzzy pricing of binary option based on the long memory property of financial markets. J Intell Fuzzy Syst 38:4889-4900

Tan X, Li S, Wang S (2020) Pricing European-style options in general Levy process with stochastic interest rate. Mathematics 8(5):731

Thiagarajah K, Appadoo SS, Thavaneswaran A (2007) Option valuation model with adaptive fuzzy numbers. Comput Math Appl 53:831-841

Tolga AC (2017) New product development process valuation using compound options with type-2 fuzzy numbers. Proc Int MultiConference Eng Comput Scientists 2:1-6

Wang X, Hea J (2016) A geometric Levy model for n-fold compound option pricing in a fuzzy framework. J Comput Appl Math 306:248-264

Wu H-C (2004) Pricing European options based on the fuzzy pattern of Black-Scholes formula. Comput Operat Res 31:1069-1081

Wu L, Wang J-T, Liu J-F, Zhuang Y-M (2017) The total return swap pricing model under fuzzy random environments. Discrete Dyn Nat Soc 1-10:2017

Xian-Dong W, Jian-Min H (2014) Reload option pricing in fuzzy framework. pp 147-152

Xu W, Peng X, Xiao W (2013) The fuzzy jump-diffusion model to pricing European vulnerable options. Int J Fuzzy Syst 15(3):317-325
Yoshida Y (2003) The valuation of European options in uncertain environment. Eur J Operat Res 145(1):221-229

Zadeh L (1975a) The concept of a linguistic variable and its application to approximate reasoning-i. Info Sci 8(3):199-249

Zadeh L (1975b) The concept of a linguistic variable and its application to approximate reasoning-ii. Info Sci 8(4):301-357

Zadeh L (1975c) The concept of a linguistic variable and its application to approximate reasoning-iii. Info Sci 9(1):43-80

Zhang H, Watada J (2018a) A European call options pricing model using the infinite pure jump Levy process in a fuzzy environment. IEEJ Trans Electr Electron Eng 13(10):1468-1482

Zhang H, Watada J, (2018b) Building Fuzzy Variance Gamma Option Pricing Models with Jump Levy Process. In: Czarnowski I, Howlett RJ, Jain LC (eds) Intelligent decision technologies, (2017) Springer International Publishing, Cham, pp 105-116

Zhang LH, Zhang WG, Xu WJ, Xiao WL (2012) The double exponential jump diffusion model for pricing European options under fuzzy environments. Econ Modell 29(3):780-786

Zhang W-G, Xiao W-L, Kong W-T, Zhang Y (2015) Fuzzy pricing of geometric Asian options and its algorithm. Appl Soft Comput $28: 360-367$

Zmeskal Z (2010) Generalised soft binomial American real option pricing model (fuzzy-stochastic approach). Eur J Operat Res 207(2):1096-1103

Publisher's Note Springer Nature remains neutral with regard to jurisdictional claims in published maps and institutional affiliations. 